\title{
ORDER PROPERTIES OF BOUNDED OBSERVABLES
}

\author{
NEAL ZIERLER
}

Continuing the development [4] of an aspect of the approach to the axiomatization of quantum mechanics of G. W. Mackey [3], we consider here the real linear space $X$ of signed measures on the set $P$ of events generated by the states, and the set $Y_{0}$ of linear functionals on $X$ which are induced in a natural way by the bounded observables. A necessary and sufficient condition for two events to be simultaneously measurable is found in terms of the order structure of $Y_{0}$, with the following consequence: if $Y_{0}$ is a lattice, $P$ is deterministic. At the opposite extreme, $Y_{0}$ is said to be an "anti-lattice" 1 if the greatest lower bound exists only for comparable pairs of its elements and we show in this case that the center of $P$ is trivial.

Our results extend those of R. V. Kadison [1], in which $Y_{0}$ and $P$ are the self-adjoint operators and projections respectively in a uniformly closed self-adjoint operator algebra. While the framework and plan of the proofs were inspired by Kadison's work, almost none of the apparatus used by him is available here with the result that, in detail, our techniques are quite different from his.

Let $P$ be a weakly modular partially ordered set (see [4]). A function $x$ from $P$ to the non-negative real numbers and $+\infty$ is said to be a measure if $x(0)=0$ and $x$ is countably additive in the sense that whenever $\left\{a_{i}\right\}$ is a pairwise orthogonal sequence of elements of $P$, then $x\left(\cup a_{i}\right)=\sum x\left(a_{i}\right)$. If $x$ is a measure and $\left\{b_{i}\right\} \subset P$ is an increasing (decreasing) sequence with supremum (infimum) $b$, then $x\left(b_{i}\right)$ $\rightarrow x(b)$. A countably additive function $x$ from $P$ to the extended real numbers is a signed measure if $x(0)=0$ and $x$ takes on at most one of the values $+\infty$ and $-\infty ; x$ is finite if $x(1)$ is finite. Define the functions $s$ and $i$ on the signed measures on $P$ by $s(x)=\sup \{x(a): a \in P\}$, $i(x)=\inf \{x(a): a \in P\}$ and set $\|x\|=s(x)-i(x)$. Clearly $\|x\|<\infty$ if and only if $x$ is finite. It is easy to see that $\|x\|=\sup \left\{x(a)-x\left(a^{\prime}\right): a \in P\right\}$.

Lemma 1. Let $X$ be a real linear space of finite signed measures on $P$. Then the function \|\| defined above is a norm for $X$ and, under this norm and its natural partial ordering, $X$ is a partially ordered normed linear space. That is, ${ }^{2}$

Received by the editors October 23, 1961 and, in revised form, February 2, 1962.

1 Kadison [1].

${ }^{2}$ See $[2]$. 
(1) $x \geqq y$ and $y \geqq x$ imply $x=y$.

(2) $x \geqq y$ and $y \geqq z$ imply $x \geqq z$.

(3) $x \geqq y$ and $\lambda \geqq 0$ imply $\lambda x \geqq \lambda y$.

(4) $x \geqq y$ implies $x+z \geqq y+z$ for all $z$.

(5) $x_{n} \geqq 0,\left\|x_{n}-x\right\| \rightarrow 0$ imply $x \geqq 0$.

In addition

(6) $x \geqq 0$ and $y \geqq 0$ imply $\|x+y\|=\|x\|+\|y\|$.

Proof. Since $i(x) \leqq s(x),\|x\| \geqq 0$; clearly, $\|x\|=0$ if and only if $x=0$. If $\lambda \geqq 0,\|\lambda x\|=\lambda\|x\|$ is obvious while $\|x\|=\|-x\|$ follows from the fact that $s(-x)=-i(x)$. Finally, $s(x+y) \leqq s(x)+s(y)$ and $i(x+y)$ $\geqq i(x)+i(y)$ so $\|x+y\| \leqq\|x\|+\|y\|$, and \|\| is a norm for $X$.

Properties (1)-(4) are obvious. If $x(a)<0$ for some $a \in P,\left\|x_{n}-x\right\|$ $\geqq s\left(x_{n}-x\right) \geqq-x(a)$ since $x_{n} \geqq 0$. This contradiction of the hypothesis $\left\|x_{n}-x\right\| \rightarrow 0$ proves (5), while (6) follows at once from the fact that $\|z\|=z(1)$ for $z \geqq 0$.

A function $A$ from the set $B$ of Borel subsets of the real line $R$ to $P$ is said to be an observable if it has the following properties:

(7) $A_{\phi}=0, A_{R}=1$.

(8) If $E_{1}, E_{2}, \cdots$ are pairwise disjoint Borel sets, then $A_{E_{1}}$, $A_{E_{2}}, \cdots$ are pairwise orthogonal members of $P$ and $A_{\cup E_{i}}=\bigvee A_{E_{i}}$.

The spectrum of an observable $A$ is defined as follows. Let $N$ $=\left\{\right.$ open $\left.E \subset R: A_{E}=0\right\}$ and choose $\left\{E_{i}\right\} \subset N$ with $\cup E_{i}=\bigcup_{E \in N} E$ and $E_{1} \subset E_{2} \subset \cdots$. Then $A_{\cup E_{i}}=\bigvee A_{E_{i}}=0$ so $\bigcup E_{i}$ is the largest member of $N$ and we set spectrum $A=$ complement $\bigcup E_{i}$. Define the norm of $A,\|A\|$, as $\sup \{|\lambda|: \lambda \in$ spectrum $A\}$ and say $A$ is bounded if $\|A\|<\infty$. Let $O$, respectively $O_{b}$, denote the set of all, respectively all bounded, observables.

Every signed measure $x$ on $P$ determines a function $m_{x}$ from $O$ to the signed measures on $B: m_{x}(A)(E)=x\left(A_{E}\right)$. If $x$ is a state then $m_{x}(A)$ is clearly a probability measure on $B$ for every observable $A$. It is easy to see that $\|x\|=\sup \left\{\left\|m_{x}(A)\right\|: A \in O\right\}$.

Let $X$ be a real linear space of finite signed measures on $P$. Each observable $A$, respectively element $a$ of $P$, defines a linear functional $L_{A}$, respectively $L_{a}$, on $X$ by $L_{A}(x)=\int_{-\infty}^{\infty} \lambda d m_{x}(A)(\lambda)$, respectively $L_{a}(x)=x(a)$. Evidently, if $A$ is the observable with $A_{\{1\}}=a$ and $A_{\{0\}}=a^{\prime}$, then $L_{A}=L_{a}$.

Lemma 2. Let $x$ be a finite signed measure on $P$. Then $\|x\|$ $=\sup \left\{\left|L_{A}(x)\right|:\|A\|=1\right\}$.

Proof. Let $\lambda=\sup \left\{\left|L_{A}(x)\right|:\|A\|=1\right\}$. Clearly $\left|L_{A}(x)\right| \leqq\|x\|\|A\|$ holds for all observables $A$ so $\lambda \leqq\|x\|$. On the other hand, we may 
choose $a_{n} \in P$ so that $x\left(a_{n}\right)-x\left(a_{n}^{\prime}\right) \rightarrow\|x\|$ and let $A(n)$ denote the observable with $A(n)_{\{1\}}=a_{n}, A(n)_{\{-1\}}=a_{n}^{\prime}$. Then $\|A(n)\|=1, L_{A(n)}(x)$ $\rightarrow\|x\|$ and the result follows.

Corollary. If $A$ is a bounded observable, $L_{A}$ is a bounded linear functional on $X$ and $\left\|L_{A}\right\| \leqq\|A\|$.

Now suppose $P$ is the set of events of a system $S, P$ of states and events, ${ }^{3}$ and henceforward let $X$ denote the real linear space of signed measures on $P$ obtained as the set of all finite real linear combinations of members of $S$.

Lemma 3. For $A \in O_{b},\left\|L_{A}\right\|=A$.

Proof. Since spectrum $A$ is a closed subset of $R$, we may choose $\lambda \epsilon$ spectrum $A$ with $|\lambda|=\|A\|$. Then if $\epsilon>0$, it follows from the definition of spectrum $A$ that the event $a=A_{(\lambda-\epsilon, \lambda+\epsilon)} \neq 0$ and hence there exists $f \in S$ with $f(a)=1$. Then $\lambda-\epsilon \leqq\left|L_{A}(f)\right| \leqq \lambda+\epsilon$ and, since $\|f\|=1,\left\|L_{A}\right\|=\sup \left\{\left|L_{A}(x)\right|: x \in X,\|x\|=1\right\} \geqq\left|L_{A}(f)\right| \geqq\|A\|-\epsilon$. Thus, $\left\|L_{A}\right\| \geqq\|A\|$, and the opposite inequality is supplied by the preceding corollary.

Let $Y_{0}=\left\{L_{A}: A \in O_{b}\right\}$, let $Y_{0}^{+}$denote the set of non-negative members of $Y_{0}$ and let $Y$ denote the subspace of $X^{*}$ generated by $Y_{0}$. Note that the mapping $a \rightarrow L_{a}$ is an isomorphism of the partially ordered set $P$ in the partially ordered set $Y_{0}$.

Lemma 4. Let $T$ be a subset of $Y_{0}$ containing $0, L_{a}, L_{b}$ and $L_{a b}$ for some $a, b \in P$. Then if $L_{a}$ and $L_{b}$ have a greatest lower bound $L_{A}$ in $T$, $L_{A}=L_{a b}$.

Proof. $0 \leqq L_{a}, L_{b}$ implies $0 \leqq L_{A}$ and $\|A\|=\sup \left\{L_{A}(f): f \in S\right\}$ $\leqq \sup \left\{L_{a}(f): f \in S\right\} \leqq 1$. Now $f(a)=0$ implies $m_{f}(A)$ is concentrated in 0, i.e., $f\left(A_{\{0\}}\right)=1$. It follows that $A_{\{0\}}^{\prime} \leqq a$; similarly $A_{\{0\}}^{\prime} \leqq b$ so $A_{\{0\}}^{\prime} \leqq a b$. Then if $g \in S, \quad L_{A}(g)=\int_{0}^{1} \lambda d m_{g}(A)(\lambda) \leqq g\left(A_{(0,1]}\right)=g\left(A_{\{0\}}^{\prime}\right)$ $\leqq g(a b)=L_{a b}(g)$ so $L_{A} \leqq L_{a b}$. Making use now of the assumption that $A$ is the greatest lower bound of $L_{a}$ and $L_{b}$ we obtain the result and

Corollary. $L_{a b}=L_{a} \wedge L_{b}$ in $Y_{0}^{+}$.

THEOREM 1. The events $a$ and $b$ are simultaneously measurable if and only if there exists a linear subspace $W$ of $Y$ containing $L_{a}, L_{b}, L_{a \vee b}$ and $L_{a b}$ such that $L_{a} \wedge L_{b}$ exists in $W \cap Y_{0}$.

Proof. Suppose $L_{a} \wedge L_{b}=L_{A} \in W$ as in the statement. Then

${ }^{3}$ See [4]; actually, the following weaker set of postulates suffices here: E1, E2, E3, E5, S2 and the following: if $f(a)=1$ whenever $f(b)=1$, then $b \leqq a$. 


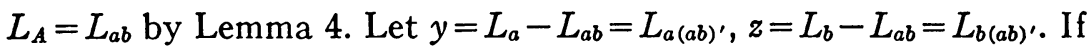
$w \in W$ with $w \leqq y, z$ then $w+L_{a b} \leqq L_{a}, L_{b}$ so $w+L_{a b} \leqq L_{a b}$ and $w \leqq 0$. It follows that $y \wedge z=0$ in $W$. Let $u=L_{a \vee b}-z=L_{(a \vee b)\left(b^{\prime} \vee a b\right)}$; then $y=L_{a(a b)^{\prime}}$ $\leqq L_{a \vee b}=u+z$ so $y-u \leqq z$. Since $0 \leqq u, y-u \leqq y$ and hence $y-u \leqq y \wedge z$ $=0$, i.e., $y \leqq u$. Thus, $a(a b)^{\prime} \leqq(a \bigvee b)\left(b^{\prime} \bigvee a b\right) \leqq b^{\prime} \bigvee a b$. Joining $b^{\prime} \bigvee a b$ to both ends of the inequality gives $a(a b)^{\prime} \bigvee a b \bigvee b^{\prime}=a \bigvee b^{\prime} \leqq b^{\prime} \bigvee a b$. Since the opposite inequality is trivial, we have, taking orthocomplements, $b a^{\prime}=b(a b)^{\prime}$. Hence $b=b(a b)^{\prime} \bigvee b a=b a^{\prime} \bigvee b a$ and the result follows from [4, Lemma 1.4].

Conversely, if $a$ and $b$ are simultaneously measurable, the subspace $W$ of $Y$ generated by the mutually exclusive events $a b, a b^{\prime}, a^{\prime} b$ and $a^{\prime} b^{\prime}$ is clearly a lattice contained in $Y_{0}$.

Corollary. If $Y_{0}$ is a lattice, then $P$ is deterministic.

Proof. Suppose $Y_{0}$ is a lattice, let $a$ and $b$ be events and let $W$ be the subspace of $Y$ generated by $L_{a}, L_{b}, L_{a \vee b}$ and $L_{a b}$. Then $L_{a b}$ $=L_{a} \wedge L_{b}$ in $Y_{0}$ by Lemma 4 and the hypothesis so, a fortiori, $L_{a b}$ $=L_{a} \wedge L_{b}$ in $W \cap Y_{0}$. Hence $a$ and $b$ are simultaneously measurable by the theorem and $P$ is deterministic.

If $A \in O$ and $\alpha$ is a Borel function from $R$ to $R$, the observable $C$ such that $C_{E}=A_{\alpha(E)}^{-1}$ for all Borel sets $E$ is clearly unique and is denoted $\alpha(A)$. Observables $A$ and $B$ are simultaneously measurable or commute if there exist an observable $C$ and Borel functions $\alpha$ and $\beta$ from $R$ to $R$ such that $A=\alpha(C)$ and $B=\beta(C)$.

Lemma 5. Let $A$ and $B$ be commuting bounded observables. Then $L_{A} \leqq L_{B}$ if and only if whenever $\alpha, \beta$ and $C$ are two Borel functions and $a$ bounded observable respectively such that $A=\alpha(C)$ and $B=\beta(C)$, $\alpha(\lambda) \leqq \beta(\lambda)$ holds for almost all $\lambda$ relative to $m_{f}(C)$ for all $f \in S$.

Proof. Suppose there exists $f \in S$ and a Borel set $E$ of positive $m_{f}(C)$ measure such that $\beta(\lambda)<\alpha(\lambda)$ on $E$. Since $m_{f}(C)(E)=f\left(C_{E}\right)>0$, $C_{E} \neq 0$ so $g\left(C_{E}\right)=1$ for some $g \in S$. But then $L_{B}(g)=\int_{E} \beta(\lambda) d m_{g}(C)(\lambda)$ $<\int_{E} \alpha(\lambda) d m_{g}(C)(\lambda)=L_{A}(g)$, which proves the nontrivial half of the lemma.

Corollary. Let $A$ and $B$ be commuting bounded observables and suppose that $L_{A} \leqq L_{B}$. Then $\int_{E} \lambda d m_{f}(A)(\lambda) \leqq \int_{E} \lambda d m_{f}(B)(\lambda)$ for all $f \in S$ and Borel sets $E$.

Proof. Suppose, on the contrary, that there exist $f, E$ such that $\int_{E} \lambda d m_{f}(A)(\lambda)>\int_{E} \lambda d m_{f}(B)(\lambda)$. But then if $\alpha, \beta$ and $C$ are as in Lemma 5, $\int_{E} \alpha(\lambda) d m_{f}(C)(\lambda)>\int_{E} \beta(\lambda) d m_{f}(C)(\lambda)$ and so $\alpha(\lambda)>\beta(\lambda)$ must hold on some Borel subset of $E$ of positive $m_{f}(C)$ measure. 
LemMa 6. Let $A$ and $B$ be commuting bounded observables and let $W=\left\{L_{C}: C \in O_{b}, C\right.$ commutes with $A$ and $\left.B\right\}$. Then $L_{A}$ and $L_{B}$ have a supremum and an infimum in $W$.

Proof. Let $\alpha$ and $\beta$ be Borel functions and $C \in O_{b}$ such that $A=\alpha(C), B=\beta(C)$ and let $D=(\sup \{\alpha, \beta\})(C)$. Obviously, $D \in W$, and $L_{D} \geqq L_{A}, L_{B}$ by Lemma 5 . Now let $D_{1}$ be any member of $W$ such that $L_{D_{1}} \geqq L_{A}, L_{B}$, let $E_{\alpha}=\{\lambda: \alpha(\lambda) \geqq \beta(\lambda)\}$ and let $E_{\beta}$ $=\{\lambda: \alpha(\lambda)<\beta(\lambda)\}$. Then, by Lemma 5, Corollary,

$$
\begin{aligned}
& \int_{E_{\boldsymbol{\alpha}}} \lambda d m_{f}\left(D_{1}\right)(\lambda) \geqq \int_{E_{\boldsymbol{\alpha}}} \lambda d m_{f}(A)(\lambda) \text { and } \\
& \int_{E_{\beta}} \lambda d m_{f}\left(D_{1}\right)(\lambda) \geqq \int_{E_{\beta}} \lambda d m_{f}(B)(\lambda) \quad \text { for all } f \in S .
\end{aligned}
$$

Hence

$$
\begin{aligned}
L_{D_{1}}(f) & =\int \lambda d m_{f}\left(D_{1}\right)(\lambda) \geqq \int_{E_{\alpha}} \lambda d m_{f}(A)(\lambda)+\int_{E_{\beta}} \lambda d m_{f}(B)(\lambda) \\
& =\int_{E_{\alpha}} \alpha(\lambda) d m_{f}(C)(\lambda)+\int_{E_{\beta}} \beta(\lambda) d m_{f}(C)(\lambda) \\
& =\int \sup \{\alpha, \beta\}(\lambda) d m_{f}(C)(\lambda)=L_{D}(\lambda)
\end{aligned}
$$

and so $L_{D}=L_{A} \bigvee L_{B}$ in $W$. Similarly, $L_{(\inf \{\alpha, \beta\})(C)}=L_{A} \wedge L_{B}$ in $W$.

Lemma 7. Suppose a belongs to the center $\mathcal{C}$ of $P$ and define the observable $A$ by $A_{\{1\}}=a, A_{\{0\}}=a^{\prime}$. Then $A$ belongs to the center of $O_{b}$.

Proof. Let $B \in O_{b}$ and define a function $C$ from the Borel subsets of the real line to $P$ as follows: $C_{E}=a B_{E} \vee a^{\prime} B_{E-3|| B||}$. Clearly $C \phi=0$ and $C_{R}=1$. If $E$ and $F$ are disjoint Borel sets, $a B_{E} \perp a B_{F}$ since $B_{E} \perp B_{F}$ while $a B_{E} \perp a^{\prime} B_{F-3|| B||}$ since $a \perp a^{\prime}$; similarly, $a^{\prime} B_{E-3|| B||}$ is orthogonal to $a B_{F}$ and $a B_{F-3|| B||}$ so $C_{E} \perp C_{F}$. If $E_{1}, E_{2}, \cdots$ are pairwise disjoint, then

$$
\begin{aligned}
\bigvee C_{E_{i}} & =\bigvee\left(a B_{E_{i}} \bigvee a^{\prime} B_{E_{i}-3|| B||}\right)=\bigvee a B_{E_{i}} \bigvee \bigvee a^{\prime} B_{E_{i}-3|| B \|} \\
& =a \bigvee B_{E_{i}} \bigvee a^{\prime} \bigvee B_{E_{i}-3\|B\|}=a B \cup_{E_{i}} \bigvee a^{\prime} B \cup\left(E_{i}-3\|B\|\right) \\
& =a B \cup E_{i} \bigvee a^{\prime} B \cup_{E_{i}-3\|B\|}=C \cup_{E_{i}}
\end{aligned}
$$

since $a \in \mathfrak{C}$ and $B \in O_{b}$ and it follows that $C \in O_{b}$. Let $\alpha$ be the characteristic function of the set $-\|B\| \leqq \lambda \leqq\|B\|$ and let $\beta$ be a Borel function on the line such that $\beta(\lambda)=\lambda$ for $-\|B\| \leqq \lambda \leqq\|B\|$ and $\beta(\lambda)$ 
$=\lambda-3\|B\|$ for $2\|B\| \leqq \lambda \leqq 4\|B\|$. Then if $E \subset[-\|B\|,\|B\|],(\beta(C))_{E}$ $=C_{\beta^{-1} E}=a B_{\beta^{-1} E} \bigvee a^{\prime} B_{\beta^{-1} E-3\|\mid B\|}=a B_{E} \bigvee a^{\prime} B_{E}=B_{E}$, i.e., $\beta(C)=B$. Evidently, $\alpha(C)=A$ and so $A$ and $B$ commute; since $B \in O_{b}$ was arbitrary, $A \in$ center $O_{b}$ as was to be proved.

Lemma 8. Suppose $A$ and $B$ are commuting observables and $\gamma$ and $\delta$ are Borel functions on the line. Then $\gamma(A)$ and $\delta(B)$ commute.

Proof. If $A=\alpha(C)$ and $B=\beta(C)$ then $\gamma(A)=\gamma \circ \alpha(C)$ and $\delta(B)$ $=\delta \circ \beta(C)$.

A partially ordered set is said to be an anti-lattice if suprema and infima exist only for comparable pairs of its elements. The Corollary of Theorem 1 asserts that if $Y_{0}$ is a lattice, $\mathfrak{e}=P$. The following theorem provides the corresponding conclusion for the opposite extreme.

Theorem 2. If $Y_{0}$ is an anti-lattice, then $\mathfrak{e}=\{0,1\}$.

Proof. Suppose, on the contrary, that there exists $a \in \mathfrak{C}$ with $0<a<1$ and let $A$ and $I$ be the observables defined as follows: $A_{\{1\}}=a, A_{\{0\}}=a^{\prime}, I_{\{1\}}=1$. Then $A$ and $I$ belong to the center of $O_{b}$ by Lemma 7 and $2 A \in$ center $O_{b}$ also by Lemma 8 . Hence $L_{2 A}$ and $L_{I}$ have a supremum and an infimum in $Y_{0}$ by Lemma 6 and it follows from the hypothesis that $L_{2 A}$ and $L_{I}$ are comparable. But by $S 2$ we can find $f, g$ in $S$ such that $f(a)=1$ and $g(a)=0$. Then $L_{2 A}(f)$ $=2>1=L_{I}(f)$ while $L_{2 A}(g)=0<1=L_{I}(g)$ and this contradiction shows that $0<a<1$ is incompatible with $A \in \mathcal{C}$.

\section{REFERENCES}

1. R. V. Kadison, Order properties of bounded, self-adjoint operators, Proc. Amer. Math. Soc. 2 (1951), 505-510.

2. S. Kakutani, Concrete representation of abstract $(L)$-spaces and the mean ergodic theorem, Ann. of Math. (2) 42 (1941), 523-537.

3. G. W. Mackey, Quantum mechanics and Hilbert space, Amer. Math. Monthly 64, No. 8, Part II (1957), 45-57.

4. N. Zierler, Axioms for non-relativistic quantum mechanics, Pacific J. Math. 2 (1961), 1151-1169.

Mitre Corporation Lincoln Laboratory 